\title{
¿Para qué enseñar español en educación primaria?
}

(What Is the Objective in Teaching Spanish in Elementary Education?)

\author{
Mirián Adriana Noriega JaCOB ${ }^{\circledR}$, Centro Regional de Formación Docente e \\ Investigación Educativa de Sonora, Sonora, México
}

\section{Volumen 2, número 1}

Junio 2017

p. $72-93$

Este número se publicó el 30 de junio de 2017

Artículo recibido: 17 de febrero de 2017

Artículo aprobado: 1 de junio 2017

ISSN: 2448-5942, doi: https://doi.org/10.36799/el.v2i1.52

\section{Cómo citar:}

Noriega Jacob , M. A. (2017). ¿Para qué enseñar español en educación primaria?. Estudios $\lambda a m b d a$. Teoría y práctica de la didáctica en lengua y literatura. , 2(1), 72-93. https://doi.org/10.36799/el.v2i1.52

Derechos de autor: El autor o autores conservan en todo momento sus derechos morales y patrimoniales sobre la obra; la obra no se puede alterar, transformar o ampliar; siempre debe reconocerse la autoría del documento referido. Ninguna de las modalidades de los documentos publicados en Estudios Aambda. Teoría y práctica de la didáctica en lengua y literatura tienen fines comerciales de naturaleza alguna.

Los contenidos de este artículo están bajo una licencia de Creative Commons Atribución No Comercial- Sin Derivadas 4.0 Internacional (c) (1) 


\title{
¿Para qué enseñar español en educación primaria?
}

\author{
What Is the Reason in Teaching Spanish in Elementary Education?
}

\author{
Mirián AdRIANA NORIEGA JACOB ${ }^{1}$
}

\begin{abstract}
Resumen
Las prácticas docentes parten de los conocimientos disciplinar, didáctico y curricular; sin alguno de estos tres conocimientos, el proceso de enseñanza presenta dificultades para un logro satisfactorio de aprendizajes significativos y claves para el estudiante. La claridad que tiene el docente sobre la declaración de los propósitos curriculares permite obtener un proceso de enseñanza y de aprendizaje exitoso, pues a partir de esto el docente diseña intervenciones didácticas de manera efectiva para el dominio disciplinar que los estudiantes deberán lograr en su proceso de aprendizaje. El presente texto tiene como objetivo mostrar una exploración sobre los conocimientos de los propósitos de la enseñanza del español en educación primaria que tiene un grupo de 40 profesores de diferentes ciudades del estado de Sonora. La metodología empleada de esta investigación se caracteriza por ser cualitativa, puesto que los datos analizados parten de la redacción libre sobre los propósitos de la enseñanza del español en educación primaria. Los resultados de esta exploración muestran que para el docente la enseñanza del español tiene como objetivo que el niño aprenda a leer, escribir y hablar, esto sin incluir intenciones socio-comunicativas que el niño necesita al interactuar con la sociedad. Sin embargo, la discusión de este artículo se centra en la postura sobre la enseñanza del español como un espacio que promueve distintos análisis y reflexiones sobre las características y el uso de la lengua que le permiten al niño interactuar dentro de una sociedad a través de recursos lingüísticos.
\end{abstract}

PALABRAS CLAVES: lectura, escritura, educación primaria, propósitos.

\begin{abstract}
The teaching practices start from knowledge of the discipline, didactics and the curricular; without any of these three aspects, the education process present difficulties for a satisfactory achievement of significant and key learning for the student. The clarity that the teacher has on the purpose of the curricular aims will allow the student to obtain a successful process of education and learning. From this point on the teacher will design effective didactic interventions for the disciplinary mastering that the students will have to manage in their learning process. The Aim of this text is to show an exploration on the awareness about the purposes of teaching Spanish in elementary education, awareness that has a group of 40 teachers from different cities of the state of Sonora, Mexico. The methodology used for this investigation is qualitative, since the analyzed information departs from open questions. The results of this research shows that teaching of Spanish has as a target the child being able to read, write and speak, besides the social communicative skills that he/she will require when interacting in society. However, the discussion of this article remains open on the premise that teaching a language, in this case Spanish, promotes different analysis and reflections over the characteristics and the use of the language that permit the child to interact within a society.
\end{abstract}

KEY WORDS: Reading, writing, elementary education, purposes.

${ }^{1}$ Licenciada en Lingüística por la Universidad de Sonora, Maestra en Educación Basada en Competencias por la Universidad del Valle de México y Doctora en Humanidades por la Universidad de Sonora. Actualmente se desempeña profesionalmente en México como profesora-investigadora de la División de Lenguaje del Centro Regional de Formación Docente e Investigación Educativa del Estado de Sonora en México. Correo electrónico: mnoriega@crfdies.edu.mx y noriega adriana@hotmail.com

Artículo recibido: 17 de febrero de 2017

Artículo enviado a corrección: 17 de abril de 2017

Aprobado: 1 de junio 2017

Noriega Jacob doi: https://doi.org/10.36799/el.v2i1.52 Volumen 2, número 1, Año 2017, ISSN: 2448-5942 


\section{INTRODUCCIÓN}

Las políticas educativas mexicanas han puesto la atención en las evaluaciones del contenido, las habilidades y las prácticas que contribuyen al desarrollo cognitivo de los niños, así como, el conocimiento y las competencias didácticas de los docentes de Educación Básica; evaluaciones que han permitido generar líneas de acciones nacionales en búsqueda de mejora para la educación. El Plan Nacional para la Evaluación de los Aprendizajes (PLANEA) evalúa los aprendizajes claves de dos campos de formación, el primer Pensamiento Matemático y el segundo Lenguaje y Comunicación. Particularmente en este último, evalúa sobre las habilidades de comprensión de textos de los niños, dejando de lado las demás competencias lingüísticas que pretende lograr la Educación Básica con lo cual promovería de una manera indirecta a la concentración de esfuerzos a la enseñanza de las habilidades evaluadas por parte de los docentes con el fin de reflejar avances significativos de sus alumnos en el campo evaluado. A pesar de la delimitación de la evaluación de los campos de formación, los resultados muestran grandes retos educativos para la educación primaria del Estado de Sonora, esto ya que según los datos de la Prueba PLANEA 2015 publicados por la Secretaría de Educación Pública [SEP] (2017) el niño sonorense termina la primaria con habilidades que se encuentran por debajo de la media nacional.

Por otra parte, las evaluaciones realizadas a los profesores mexicanos han sido a través del Sistema Nacional de Registro del Servicio Profesional Docente, evaluaciones que permiten al docente ingresar, lograr promoción y permanecer dentro de dicho sistema. De manera específica el proceso de la evaluación de permanencia docente se realiza con: 1) un informe de cumplimiento de responsabilidades profesionales, 2) expediente de evidencias de enseñanza, 3) examen de conocimientos y competencias didácticas, 4) planeación didáctica argumentada, y 5) examen complementario en el que se evalúa competencias lingüísticas del inglés como segunda lengua.

Si bien es cierto que la evaluación docente incluye diversos aspectos, no explora sobre el conocimiento disciplinar requerido para la enseñanza de las asignaturas del programa y saberes requeridos para la implementación del plan y programa curricular de Educación Básica. Considerando lo anterior, el impacto de la enseñanza del español en educación primaria tiene 
como principal referente de diagnóstico los resultados obtenidos de los aprendizajes claves de los niños a través del examen de PLANEA 2015 (SEP, 2017).

El conocimiento disciplinar y la profundización del conocimiento del programa de español son parte de bases fundamentales para la práctica docente de la enseñanza de la lengua que se concreta en los niños con el logro de aprendizajes significativos. El conocimiento del programa de español es una herramienta que guía las directrices de la educación con las que se materializa las políticas educativas. Ante los bajos resultados obtenidos con los niños sonorenses de educación primaria en la Prueba PLANEA 2015 (SEP, 2017) surge la interrogante sobre la precisión y claridad que tienen los docentes en relación a los propósitos de la enseñanza del español en educación primaria, es decir, ¿para qué el docente enseña español a los niños? El objetivo principal de este texto es mostrar una exploración sobre los conocimientos de los propósitos de la enseñanza del español en educación primaria que tiene un grupo de docentes; conocimientos que no se exploran en las evaluaciones nacionales, aplicadas hasta el año 2016, y que permitirían proponer líneas de acciones para el fortalecimiento de la práctica docente en la enseñanza del español.

La escuela ha tenido una participación importante en las prácticas lingüísticas de los miembros de culturas escritas, incluso, la enseñanza y el aprendizaje de la lengua en el contexto áulico de estas culturas parecieran procesos naturales del ser humano. Sin embargo, los procesos de enseñanza y aprendizaje de la lengua dentro de situaciones escolarizadas requieren la declaración de los objetivos de enseñanza y aprendizaje que permita la claridad de la finalidad de dichos procesos, sin ello, cabría la posibilidad de retroceso educativo de estos grupos sociales.

La escuela tiene gran influencia en el desarrollo de la sociedad que paulatinamente se materializa en cambios sociales; la declaración de los objetivos de enseñanza y aprendizaje, así como la claridad de los mismos, son fundamentales, puesto que sin ellos no tendría sentido los procesos de enseñanza y aprendizaje, y cabría la posibilidad de retroceso educativo (Cassany et al., 2007).

Tradicionalmente la enseñanza y el aprendizaje de la lengua en el contexto áulico preparaban a los individuos para la codificación y descodificación de la lengua. No obstante, en 
la actualidad escribir y leer son actividades que van más allá de codificar y descodificar, por lo cual se requiere de una conciencia y múltiples reflexiones metalingüísticas en ambos procesos.

Según Fons (2007: 22) "escribir es el proceso mediante el cual se produce el texto escrito"; proceso que necesita considerar al posible lector del texto, el mensaje y la manera de transmitirlo discursivamente. Además, el proceso de composición de textos se realiza (o debiera realizarse) a través de tres subprocesos no necesariamente lineales; el primero es la planificación, en el cual el autor de un escrito toma decisiones sobre su producción, esto puede ser antes o mientras escribe. El segundo subproceso es la textualización, en él se construye a través de unidades lingüísticas el mensaje de un escrito. Finalmente, el tercer subproceso es la revisión del texto, éste consiste en cambiar el texto con la finalidad de lograr una claridad en el mensaje a través de la supresión, sustitución, adición y/o cambio de orden de ideas y estructuras lingüísticas (Fons, 2007: 23-24).

Por otra parte, la conceptualización actual de leer implica que el individuo logre la comprensión de un conjunto de grafías y/o gráficos, con ello ya no es suficiente la descodificación de la lengua escrita. De esta manera, la lectura es un proceso que requiere determinar un objetivo con el que el lector se apoye antes, durante y después de la actividad; interactuar con el texto utilizando los conocimientos previos para transformarlos a nuevos; realizar predicciones e interpretaciones de lo que se presenta en los textos que lee; analizar, reflexionar y evaluar críticamente los contenidos de los textos según los propósitos de lectura que se establezcan.

Es decir, la lectura y la escritura son procesos en los que el individuo debe estar consciente y activo para el uso de sus conocimientos lingüísticos y cognitivos. En ambas actividades es importante que se planteen propósitos y estrategias que le permitan al sujeto producir y comprender textos. En la sociedad que tiene cultura escrita, los niños aprenden a leer y a escribir independientemente del método que se utilice, sin embargo, el niño necesita de apoyo con estrategias didácticas para aprender a organizar el texto, escribir autónomamente, analizar, evaluar, predecir, entre otras habilidades relacionadas (Rebazo y Moreno, 2005).

El estudio y el aprendizaje de la lengua en contexto escolarizado constituyen la adquisición de conocimientos culturales y de un sistema que ordena al pensamiento y la mente,

Noriega Jacob doi: https://doi.org/10.36799/el.v2i1.52 Volumen 2, número 1, Año 2017, ISSN: 2448-5942 
con estos procesos el ser humano facilita y amplía sus alternativas de comunicación (Cassany et al., 2007: 36-37).

El propósito de la enseñanza y del aprendizaje de la lengua del español en el contexto escolar implica desarrollar habilidades de pensamiento crítico y creativo en los individuos para que se comuniquen de manera eficaz en los diversos contextos sociales independientemente de su procedencia cultural. En la bibliografía consultada se plantean cuatro niveles de adquisición y dominio de la lengua escrita, el primero es el denominado ejecutivo, en él se evidencia la capacidad de uso de la lengua oral y escrita con la producción de mensajes que parten de la oralidad y viceversa, de la escritura a la oralidad; el segundo es de carácter funcional, en el cual el individuo resuelve situaciones ordinarias con el uso y la producción de los diferentes tipos de textos; el tercero es de carácter instrumental, implica que el sujeto emplee los textos para buscar y usar la información escrita; finalmente, el nivel epistémico muestra la capacidad de uso de la lengua escrita para el desarrollo del pensamiento creativo y crítico.

La enseñanza de la lengua significa promover aprendizajes significativos para el desarrollo y la interacción en grupos socioculturales estructurados donde interactúan decenas de personas alfabetizadas. El aprendizaje y el uso de la lengua escrita debe aspirar a la formación de individuos pensadores, creativos y críticos; de este modo, lo óptimo es la que la alfabetización se materialice en actividades y situaciones donde el individuo se desempeñe en un nivel epistémico.

En México, la enseñanza del español en la educación primaria tiene por propósitos ${ }^{2}$ principales que el niño se apropie de las prácticas sociales del lenguaje (individuales y colectivas), reflexione sobre las características y funciones de la lengua oral y escrita, y en el caso de la lectura que el niño logre lecturas autónomas con diversos propósitos.

Los propósitos de la enseñanza del español en educación básica son que los niños:

\footnotetext{
${ }^{2}$ Cabe señalar que los programas de los distintos grados de educación primaria se conforman por la misma información declarada para los propósitos de la enseñanza del español en educación básica, los propósitos de la enseñanza del español en primaria y el papel del docente y trabajo en el aula. Considerando lo anterior y que en la recolección de datos para la investigación los docentes se encontraban impartiendo tercer grado de primaria, el documento que se utiliza como referencia es el Programa de estudios 2011. Guia para el maestro. Educación Básica. Primaria. Tercer grado. México: SEP, 2011.
}

Noriega Jacob doi: https://doi.org/10.36799/el.v2i1.52 Volumen 2, número 1, Año 2017, ISSN: $2448-5942$ 
1) Utilicen eficientemente el lenguaje para organizar su pensamiento y su discurso; analicen y resuelvan problemas de la vida cotidiana; accedan y participen en las distintas expresiones culturales.

2) Logren desempeñarse con eficacia en diversas prácticas sociales del lenguaje y participen de manera activa en la vida escolar y extraescolar.

3) Sean capaces de leer, comprender, emplear, reflexionar e interesarse en diversos tipos de texto, con el fin de ampliar sus conocimientos y lograr sus objetivos personales.

4) Reconozcan la importancia del lenguaje para la construcción del conocimiento y de los valores culturales, y desarrollen una actitud analítica y responsable ante los problemas que afectan al mundo (SEP, 2011).

Específicamente en educación primaria con la enseñanza del español se espera que los niños:

1) Participen eficientemente en diversas situaciones de comunicación oral.

2) Lean comprensivamente diversos tipos de texto para satisfacer sus necesidades de información y conocimiento.

3) Participen en la producción original de diversos tipos de texto escrito.

4) Reflexionen de manera consistente sobre las características, el funcionamiento y el uso del sistema de escritura (aspectos gráficos, ortográficos, de puntuación y morfosintácticos).

5) Conozcan y valoren la diversidad lingüística y cultural de los pueblos de nuestro país.

6) Identifiquen, analicen y disfruten textos de diversos géneros literarios (SEP, 2011).

Asimismo para lograr lo anterior, el papel del docente y el trabajo en el aula debe:

1) Promover el trabajo de reflexión y análisis de los textos por parte de los alumnos, plantear preguntas o hacer aseveraciones que les permitan identificar algún aspecto de lo que leen o cómo leen; alentar a los alumnos a dar explicaciones; retarlos a lograr apreciaciones 
cada vez más objetivas a través de la confrontación con los propios textos o con opiniones de sus compañeros.

2) Mostrar a los alumnos las estrategias que usa un lector o escritor experimentado, con el fin de hacer evidentes las decisiones y opciones que se presentan al desarrollar estas actividades. En este sentido, el docente es un referente para los alumnos y les facilita la relación con la lengua escrita.

3) Dar a los alumnos orientaciones puntuales para la escritura y la lectura. Ayudarlos a centrarse en las etapas particulares de la producción de textos, tales como la planeación y corrección, y a usar estrategias específicas durante la lectura, por ejemplo, realizar inferencias, crear o comprobar hipótesis, entre otras.

4) Fomentar y aprovechar la diversidad de opiniones que ofrece el trabajo colectivo y equilibrarlo con el trabajo individual, lo que da oportunidad para que los alumnos logren el máximo aprendizaje posible.

5) Estimular a los alumnos a escribir y leer de manera independiente sin descuidar la calidad de su trabajo (SEP, 2011).

De acuerdo con el Programa de Estudio (SEP, 2011) y Fons (2007), en la actualidad la enseñanza y el aprendizaje de la lectura y la escritura no deben ser considerados como procesos sumativos, sino deben ir en función de reorganizaciones de conocimiento de diferente tipo y nivel donde el niño logre aprendizajes significativos en situaciones comunicativas reales con la lengua escrita. El aprendizaje de la lengua escrita únicamente se logra cuando el individuo requiere utilizar estrategias de comprensión y producción en situaciones reales en las que busque obtener objetivos concretos.

El proceso de enseñanza y aprendizaje de la lengua debe ser considerado como prácticas culturales con las que el individuo socializa con la comunidad. En este sentido, no es importante que el niño aprenda la lengua, sino qué hace con ella (Dubois, 2006). Las prácticas docentes de la enseñanza de la lengua deben tener como eje central el logro de objetivos sociales y prácticas culturales más amplios, es decir, “(...) el uso de la literacidad es esencialmente social, no se 
localiza únicamente en la mente de las personas o en los textos leídos o escritos sino también en la interacción interpersonal y en lo que la gente hace con estos textos" (Zabala en Cassany, 2009: 24).

\section{MÉTODO}

Los datos que se presentan en este texto son obtenidos de un grupo de 40 profesores que imparten tercer grado de educación primaria adscritos al sistema público de cinco municipios del estado de Sonora ${ }^{3}$, la participación de este grupo de docentes se deriva de una selección aleatoria y no probabilístico debido a la posibilidad de acercamiento puntual con los profesores. El grupo de maestros se caracteriza por la participación predominante de mujeres (75\%), asimismo, el 62.5\% es egresado de la Licenciatura en Educación Primaria en un periodo menor a 10 años, mismo tiempo que el 50\% de los profesores está frente a grupo. Aunque en datos de los profesores no se solicitó la edad de ellos, se observa que la mitad del grupo tiene de cero a diez años de servicio docente, lo cual indica que es un grupo joven.

Los datos obtenidos surgen de encuentros con grupos de diez profesores, sumando un total de cuatro encuentros donde participaron 40 docentes. En estos espacios ellos respondieron una encuesta que explora cuestiones del Programa de Español 2011 de educación primaria (SEP, 2011), una de estas corresponde a los propósitos de la enseñanza del español en primaria que fueron solicitados a los maestros a través de una redacción libre, esto para tener mayor cercanía a los conocimientos sobre el los propósitos. La consigna dentro de la encuesta fue "en el siguiente espacio mencione los propósitos de la enseñanza del español en educación primaria”, el espacio asignado fue de una página en blanco para la redacción libre. Cabe señalar que los profesores al atender esta consigna no tenían tiempo asignado, ellos podrían atender los otros apartados de la encuesta y regresar las veces que consideraran necesario, de tal manera que no se presentaran factores emocionales (como estrés) al responder el instrumento.

Una vez registrados los datos se realizaron categorizaciones de las respuestas utilizando un total de ocho matrices; seis de ellos corresponden a los propósitos de la enseñanza del español

\footnotetext{
${ }^{3}$ El estado de Sonora se ubica en la región noroeste de México.

Noriega Jacob doi: https://doi.org/10.36799/el.v2i1.52 Volumen 2, número 1, Año 2017, ISSN: 2448-5942
} 
en primaria (una matriz por propósito declarado en el programa), uno a los propósitos generales de la educación primaria y otro al análisis de datos clasificados como otros.

Las matrices de los propósitos de la enseñanza del español en primaria y de los propósitos generales de este nivel educativo se conforman por tres elementos, el primero corresponde al registro aleatorio de cada docente (por ejemplo: PPCAB014 ${ }^{4}$, PPGUY05), el cual se utilizó para la clasificación de respuestas que corresponderían a los propósitos de la enseñanza del español; el segundo elemento es la enunciación literal emitida por el profesor; y el tercero corresponde a los datos particulares de identificación de la matriz, tales como: número de tabla y propósito correspondiente al declarado en el programa de español. En el caso de la matriz que presenta datos sobre enunciaciones de los propósitos de la enseñanza del español en educación básica se muestran los propósitos declarados en el programa y otras respuestas ajenas enunciadas en el programa, porcentaje de entradas relacionadas con propósitos en cuestión y los datos de identificación de la matriz.

\section{RESULTADOS}

El análisis de los propósitos de la enseñanza del español en educación primaria emitidos por el grupo de profesores parte de las enunciaciones que tuvieron mayor a menor entradas relacionadas con lo declarado en al programa de estudio de español de educación primaria (SEP, 2011). El análisis finaliza con la matriz que corresponde a la Tabla 8, la cual presenta datos correspondientes a los propósitos de la enseñanza del español en educación básica y otras respuestas ajenas al programa.

La escuela, como anteriormente se menciona, es una figura que influye sustancialmente en el proceso de inclusión a las culturas escritas con el aprendizaje guiado y formal de la lengua, particularmente con la lectura y la escritura. Esto se evidencia en un gran porcentaje de enunciaciones de los profesores en el que muestran mayor atención en la lectura y la producción

\footnotetext{
${ }^{4}$ La nomenclatura utilizada para referirse a la información obtenida por los participantes de la investigación se elaboró considerando la siguiente información: el rol ( $\mathrm{P}=$ Profesor), el nivel educativo en el que se desempeña ( $\mathrm{P}=$ Primaria), código para la identificación de la localidad donde se desempeña profesionalmente (GUAY=Guaymas, $\mathrm{CAB}=\mathrm{Caborca}, \mathrm{HUA}=\mathrm{Huatabampo}$, $\mathrm{NOG}=$ Nogales y $\mathrm{HMO}=$ Hermosillo) y el número aleatorio asignado para cada docente (por ejemplo: 01); de esta manera, la nomenclatura de identificación de cada participante resultaría único, por ejemplo: PPGUAY01=Profesor de Primaria de Guaymas Número 1.
}

Noriega Jacob doi: https://doi.org/10.36799/el.v2i1.52 Volumen 2, número 1, Año 2017, ISSN: 2448-5942 
escrita de los niños de primaria como habilidades a desarrollar dentro de los principales propósitos de la enseñanza del español en este nivel educativo, ya que el 40\% del grupo de docentes emiten afirmaciones en relación a la comprensión lectora que se desea lograr en los estudiantes, sin embargo, éstas son enunciaciones generales y sin fin particular de lograr la comprensión (véase tabla 1), a excepción de un maestro que incluye la finalidad (PPHUA04).

\begin{tabular}{|c|c|}
\hline PPGUAY01 & Que los alumnos comprendan textos. \\
\hline PPGUAY04 & $\begin{array}{l}\text { Que al entrar en contacto con textos pueda comprender el mensaje de } \\
\text { manera literal e implícita. }\end{array}$ \\
\hline PPCAB01 & $\begin{array}{l}\text { Que el alumno conozca los diferentes tipos de textos, que comprendan y } \\
\text { reflexionen lo que lean. }\end{array}$ \\
\hline PPCAB04 & $\begin{array}{l}\text { Que el alumno adquiera los conocimientos, habilidades y destrezas para } \\
\text { interpretar información contenida en un texto a través de la reflexión y } \\
\text { análisis. }\end{array}$ \\
\hline PPCAB05 & $\begin{array}{l}\text { Que sea capaz de interpretar y redactar textos de diferentes modalidades, } \\
\text { dependiendo del uso que desea darle. }\end{array}$ \\
\hline PPCAB06 & $\begin{array}{l}\text { Algo muy importante que comprenda lo que lea y sea capaz de dar una } \\
\text { pequeña explicación o descripción de lo leído. }\end{array}$ \\
\hline PPHUA01 & Que el alumno elabore escritos, comprenda e interprete textos. \\
\hline PPHUA02 & Lograr una lectura de comprensión. \\
\hline PPHUA04 & $\begin{array}{l}\text { Utilicen, analicen, lean, exploren, comprendan diversos tipos de textos para } \\
\text { lograr su aprendizaje y objetivos/metas individuales o personales. }\end{array}$ \\
\hline PPHUA05 & Comprensión lectora. \\
\hline PPNOG02 & Habilidad de lectura y comprensión lectora. \\
\hline PPHMO01 & $\begin{array}{l}\text { Sean capaces de leer, comprender, ampliar, reflexionar o interesarse en } \\
\text { diferentes tipos de textos. }\end{array}$ \\
\hline PPHMO02 & $\begin{array}{l}\text { Que el alumno comprenda, reflexione, analice los diferentes tipos de texto y } \\
\text { enseñanza. }\end{array}$ \\
\hline PPHMO02 & El principal propósito es la comprensión al momento de leer un texto. \\
\hline PPNOG03 & Análisis, interpretación y producción de textos. \\
\hline PPNOG05 & El alumno comprenda lo que lee en diversas fuentes de información. \\
\hline PPNGO07 & La comprensión y la expresión de textos de interés del alumno. \\
\hline
\end{tabular}

Tabla 1. Propósito de la enseñanza del español en primaria: lograr que los estudiantes "lean comprensivamente diversos tipos de texto para satisfacer sus necesidades de información y conocimiento” (SEP, 2011) 
Algo similar sucede con las afirmaciones de la producción escrita de los niños, el mismo porcentaje de los profesores (40\%) mencionan que el niño en educación primaria escribirá pero sin algún propósito comunicativo (véase tabla 2), únicamente dos docentes (PPGUAY08, PPNOG09) emite la intencionalidad de la enseñanza de la producción escrita.

\begin{tabular}{|l|l|}
\hline PPGUAY03 & $\begin{array}{l}\text { Que el alumno realice producción de textos, realice borradores, antes de } \\
\text { llegar a la producción final de un texto. }\end{array}$ \\
\hline PPGUAY04 & $\begin{array}{l}\text { Que al redactar textos pueda mandar un mensaje claro al receptor o público } \\
\text { al que va dirigido, utilizando un lenguaje acorde a los lectores. }\end{array}$ \\
\hline PPGUAY08 & Que el alumno escriba diferentes tipos de textos. \\
\hline PPCAB03 & $\begin{array}{l}\text { Que el alumno redacte, corrija y divulgue diversos tipos de textos con la } \\
\text { intención de adquirir competencias para comunicarse. }\end{array}$ \\
\hline PPCAB04 & Sea capaz de elaborar sus propios textos. \\
\hline PPCAB09 & $\begin{array}{l}\text { Que el alumno utilice su lengua materna de forma oral y escrita de la mejor } \\
\text { manera, cuidando ortografía y cuestiones gramaticales. }\end{array}$ \\
\hline PPCAB09 & Produzca diferentes tipos de textos. \\
\hline PPHUA01 & Que el alumno elabore escritos, comprenda e interprete textos. \\
\hline PPHUA03 & Creación de textos. \\
\hline PPNOG01 & Producción de textos. \\
\hline PPHMO01 & Participen en diferentes producciones de textos escritos. \\
\hline PPHMO05 & Pueda producir textos escritos. \\
\hline PPHMO06 & Producción de texto escrito. \\
\hline PPHMO08 & Que el alumno lea, escriba y redacte diferentes textos en su vida cotidiana. \\
\hline PPHMO08 & $\begin{array}{l}\text { Que el alumno sea capaz de redactar diferentes tipos de textos para } \\
\text { solucionar problemas de la vida diaria. }\end{array}$ \\
\hline PPHMO08 & $\begin{array}{l}\text { Al redactar diferentes tipos de textos que utilice los signos de puntuación y } \\
\text { coherencia. }\end{array}$ \\
\hline PPNOG03 & $\begin{array}{l}\text { Análisis, interpretación y producción de textos. } \\
\text { Que el alumno sea capaz de producir sus propios textos desarrollando la } \\
\text { expresión oral y escrita. } \\
\text { PPNOG03 } \\
\text { de la vida diaria. }\end{array}$ \\
\hline PPNOG05 & $\begin{array}{l}\text { Que el alumno adquiera las competencias y habilidades para comunicarse } \\
\text { eficazmente de forma oral y escrita. }\end{array}$ \\
\hline
\end{tabular}

Tabla 2. Propósito de la enseñanza del español en primaria: lograr que los alumnos "participen en la producción original de diversos tipos de texto escrito” (SEP, 2011)

Noriega Jacob doi: https://doi.org/10.36799/el.v2i1.52 Volumen 2, número 1, Año 2017, ISSN: 2448-5942 
Los datos presentados en la tabla 1 y la tabla 2 muestran que los docentes omiten la intencionalidad de las prácticas de lectura y escritura. Esto es de suma importancia para el proceso de aprendizaje de la lengua escrita, puesto que la declaración de la intencionalidad de dichas prácticas favorece el desarrollo metacognitivo y metalingüístico que implica leer y escribir; sin la claridad de objetivos de lectura es fácil tener experiencias poco significativas a partir del texto (Silvestri, 2006) y sin propósitos de escritura se producen textos que resultan pocos claros para el posible lector (Cassany, 1996).

Por otra parte, el 25\% del grupo de maestros emite como propósito de enseñanza del español en educación primaria algunas enunciaciones que se aproximan a las habilidades de comunicación oral de los niños. El desarrollo de las habilidades comunicativas orales requiere considerar contextos sociales y situacionales donde el niño se desenvolvería, por ello, el programa de educación básica tiene como base las prácticas sociales del lenguaje (SEP, 2011), con las cuales pretende que la enseñanza de la lengua tenga enfoque sociocultural (Cassany, 1999); en relación a esto, una parte del 25\% del grupo de docentes (PPGUAY02, PPCAB01, PPCAB03, PPCAB05, PPCAB07) incluye en la redacción de los propósitos de la enseñanza del español aspectos sociales (véase tabla 3).

\begin{tabular}{|l|l|}
\hline PPGUAY02 & Que el alumno participe en situaciones comunicativas de manera eficiente. \\
\hline PPGUAY04 & $\begin{array}{l}\text { Lograr que el alumno se comunique de manera eficiente; de manera verbal y } \\
\text { escrita, principalmente. }\end{array}$ \\
\hline PPCAB01 & $\begin{array}{l}\text { Que logren comunicarse satisfactoriamente con la sociedad, así como ser } \\
\text { capaces de resolver las dificultades que se les presenten a lo largo de su vida. }\end{array}$ \\
\hline PPCAB03 & $\begin{array}{l}\text { Conozca el lenguaje formal e informal para comunicarse en diversos } \\
\text { contextos. }\end{array}$ \\
\hline PPCAB05 & $\begin{array}{l}\text { Que el alumno conozca y utilice el lenguaje oral y escrito para interpretar y } \\
\text { comunicarse con los demás. }\end{array}$ \\
\hline PPCAB05 & \begin{tabular}{l} 
Que sea capaz de comunicarse asertivamente con sus semejantes. \\
\hline PPCAB07
\end{tabular} $\begin{array}{l}\text { Exprese sus ideas o necesidades de una manera que logre hacerse } \\
\text { comprender. }\end{array}$ \\
\hline PPCAB07 & $\begin{array}{l}\text { Utilice el lenguaje para comunicarse eficaz en todos los ámbitos de su vida } \\
\text { cotidiana. }\end{array}$ \\
\hline PPCAB09 & $\begin{array}{l}\text { Que el alumno utilice su lengua materna de forma oral y escrita de la mejor } \\
\text { manera, cuidando ortografía y cuestiones gramaticales. }\end{array}$ \\
\hline PPHUA03 & \begin{tabular}{l} 
Comunicación efectiva. \\
\hline
\end{tabular} \\
\hline
\end{tabular}

Noriega Jacob doi: https://doi.org/10.36799/el.v2i1.52 Volumen 2, número 1, Año 2017, ISSN: 2448-5942 


\begin{tabular}{|l|l|}
\hline PPHMO05 & Se exprese de forma oral y escrita. \\
\hline PPNOG09 & $\begin{array}{l}\text { Que el alumno adquiera las competencias y habilidades para comunicarse } \\
\text { eficazmente de forma oral y escrita. }\end{array}$ \\
\hline
\end{tabular}

Tabla 3. Propósito de la enseñanza del español en primaria: lograr que los estudiantes "participen eficientemente en diversas situaciones de comunicación oral” (SEP,2011)

Dentro de los propósitos de la enseñanza del español en educación primaria se incluye, entre otras cuestiones, la reflexión del uso y el funcionamiento de la lengua escrita a través de los recursos gráficos; los datos muestran que 22.5\% de los profesores enunciaron como propósitos aspectos referentes a éste (véase tabla 4). Independientemente de la declaración de este propósito en el programa, la enseñanza y el aprendizaje de recursos gráficos de la lengua escrita (como la ortografía, los signos de puntuación y la gramática de la lengua) son objeto de observación de la sociedad de la cultura escrita, posiblemente se debe a la visibilidad de la producción escrita de los individuos a diferencia de procesos como la comprensión de textos.

\begin{tabular}{|c|c|}
\hline PPCAB03 & $\begin{array}{l}\text { Utilice los signos de puntuación en lectura y escritura para mejorar la } \\
\text { comprensión del mismo. }\end{array}$ \\
\hline PPHUA02 & Conocimiento y dominio de la gramática. \\
\hline PPHUA03 & Aspectos formales del sistema de escritura (reglas ortográficas). \\
\hline PPHUA05 & Reglas ortográficas. \\
\hline PPNOG02 & El uso de signos de puntuación. \\
\hline PPHMO08 & $\begin{array}{l}\text { Al redactar diferentes tipos de textos que utilice los signos de puntuación y } \\
\text { coherencia. }\end{array}$ \\
\hline PPNOG03 & Uso de la gramática y reglas ortográficas. \\
\hline PPNOG05 & $\begin{array}{l}\text { Reconozca la estructura de los textos que redacta (coherencia, relevancia, } \\
\text { signos de puntuación, ortografía, fonemas, etc.) }\end{array}$ \\
\hline PPNGO07 & Uso de ortografía convencional. \\
\hline
\end{tabular}

Tabla 4. Propósito de la enseñanza del español en primaria: lograr que los alumnos "reflexionen de manera consistente sobre las características, el funcionamiento y el uso del sistema de escritura (aspectos gráficos, ortográficos, de puntuación y morfosintácticos)" (SEP, 2011)

La escuela tiene un rol fundamental en el acercamiento y en el uso de diversos textos en la sociedad, gran parte de las experiencias lectoras de los mexicanos parten de situaciones académicas (CONACULTA, 2006). Dentro del programa de español de educación primaria se

Noriega Jacob doi: https://doi.org/10.36799/el.v2i1.52 Volumen 2, número 1, Año 2017, ISSN: $2448-5942$ 
establece como propósito de enseñanza que los niños "identifiquen, analicen y disfruten textos de diversos géneros literarios" (SEP, 2011), el 22.5\% del grupo de maestros encuestados declaran como propósitos elementos que se aproximan a lo declarado en el programa (véase tabla 5).

\begin{tabular}{|l|l|}
\hline PPGUAY03 & Que analice información que observa en diversos medios y la difunda. \\
\hline PPCAB04 & Identifique diferentes tipos de textos. \\
\hline PPCAB04 & Conozca los diferentes tipos de lenguaje para que valore sus culturas. \\
\hline PPCAB06 & $\begin{array}{l}\text { Los alumnos identifiquen los tipos de texto, así como a desarrollar ideas a } \\
\text { partir de la lectura de uno. }\end{array}$ \\
\hline PPHUA02 & Conocer y diferenciar los distintos tipos de textos. \\
\hline PPHUA03 & Manejo de diferentes tipos de textos. \\
\hline PPNOG02 & $\begin{array}{l}\text { El uso de material escrito, como el caso de revistas científicas y libros } \\
\text { informativos y/o científicos. }\end{array}$ \\
\hline PPHMO01 & Sean capaces de identificar, analizar y disfrutar textos diversos. \\
\hline PPNOG03 & Reconozca la importancia del uso de distintas fuentes de información. \\
\hline PPNGO07 & Análisis de diferentes tipos de textos, el reconocimiento y la utilidad. \\
\hline
\end{tabular}

Tabla 5. Propósito de la enseñanza del español en primaria: lograr que los alumnos "identifiquen, analicen y disfruten textos de diversos géneros literarios”(SEP, 2011)

El $7.5 \%$ de los profesores encuestados mencionan como parte del conjunto de propósitos de la enseñanza del español aspectos de la valoración y el conocimiento de la diversidad lingüística y cultural de México, particularmente en las enunciaciones de declaraciones de valoración (véase tabla 6).

\begin{tabular}{|l|l|}
\hline PPCAB04 & Conozca los diferentes tipos de lenguaje para que valore sus culturas. \\
\hline PPNOG03 & Valorar la diversidad lingüística. \\
\hline PPNOG04 & Valores la importancia de los valores en su cultura y aprecio a la diversidad. \\
\hline
\end{tabular}

Tabla. 6 Propósito de la enseñanza del español en primaria: lograr que los alumnos "conozcan y valoren la diversidad lingüística y cultural de los pueblos de nuestro país” (SEP, 2011)

En suma, primordialmente los profesores consideran como propósitos de la enseñanza del español en primaria las habilidades de lectura y escritura; en segundo lugar el desarrollo de 
habilidades orales; en tercero la reflexión sobre el uso y funcionamiento de la lengua, así como, la identificación, análisis y el goce de diferentes géneros literarios; y en cuarto lugar el conocimiento y valoración de la diversidad lingüística y cultural (véase tabla 7). Además de enunciaciones referidas a los propósitos de la enseñanza del español en primaria, señalan como propósitos otros que no corresponden a la enseñanza del español en educación primaria (véase tabla 7), sino a aquellos de educación básica (17.5\%) y otros ajenos a los de primaria y educación básica $(82.5 \%)$ (Véase tabla 7). Estos últimos categorizados como otros se estudian con el apoyo de la tabla 8 y tabla 9 .

\begin{tabular}{|l|lc|}
\hline $\begin{array}{l}\text { Lean comprensivamente diversos tipos de texto para satisfacer sus } \\
\text { necesidades de información y conocimiento. }\end{array}$ & $40 \%$ & $(16 / 40)$ \\
\hline $\begin{array}{l}\text { Participen en la producción original de diversos tipos de texto } \\
\text { escrito }\end{array}$ & $40 \%$ & $(16 / 40)$ \\
\hline $\begin{array}{l}\text { Participen eficientemente en diversas situaciones de comunicación } \\
\text { oral }\end{array}$ & $25 \%$ & $(10 / 40)$ \\
\hline $\begin{array}{l}\text { Reflexionen de manera consistente sobre las características, el } \\
\text { funcionamiento y el uso del sistema de escritura (aspectos gráficos, } \\
\text { ortográficos, de puntuación y morfosintácticos) }\end{array}$ & $22.5 \%$ & $(9 / 40)$ \\
\hline $\begin{array}{l}\text { Identifiquen, analicen y disfruten textos de diversos géneros } \\
\text { literarios }\end{array}$ & $22.5 \%$ & $(9 / 40)$ \\
\hline $\begin{array}{l}\text { Conozcan y valoren la diversidad lingüística y cultural de los } \\
\text { pueblos de nuestro país }\end{array}$ & $7.5 \%$ & $(3 / 40)$ \\
\hline Otros & $82.5 \%$ & $(33 / 40)$ \\
\hline
\end{tabular}

Tabla 7. Porcentajes de profesores que enunciaron los propósitos de la enseñanza del español en educación primaria

En el análisis de las enunciaciones clasificada como otros en la tabla 7, se identificaron propósitos que corresponden a la educación básica. Sin embargo, el 75.75\% de las entradas como otros son ajenos a los propósitos de primaria y educación básica, lo cual indica que escasa claridad sobre la finalidad de la enseñanza del español en primaria (véase tabla 8). Estos últimos categorizados como "Enunciaciones ajenas a propósitos de la enseñanza del español en educación primaria y educación básica” son declaraciones que evidencian confusión como el rol del profesor en la enseñanza del español por propósito, así como el objeto de la enseñanza de la lengua (véase tabla 9).

Noriega Jacob doi: https://doi.org/10.36799/el.v2i1.52 Volumen 2, número 1, Año 2017, ISSN: 2448-5942 


\begin{tabular}{|l|lc|}
\hline $\begin{array}{l}\text { Enunciaciones ajenas a propósitos de la enseñanza del español en } \\
\text { educación primaria y educación básica }\end{array}$ & $75.75 \%$ & $(25 / 33)$ \\
\hline $\begin{array}{l}\text { Logren desempeñarse con eficacia en diversas prácticas sociales del } \\
\text { lenguaje y participen de manera activa en la vida escolar y } \\
\text { extraescolar. }\end{array}$ & $30.30 \%$ & $(10 / 33)$ \\
\hline $\begin{array}{l}\text { Reconozcan la importancia del lenguaje para la construcción del } \\
\text { conocimiento y de los valores culturales, y desarrollen una actitud } \\
\text { analítica y responsable ante los problemas que afectan al mundo. }\end{array}$ & $21.21 \%$ & $(7 / 33)$ \\
\hline $\begin{array}{l}\text { Utilicen eficientemente el lenguaje para organizar su pensamiento y } \\
\text { su discurso; analicen y resuelvan problemas de la vida cotidiana; } \\
\text { accedan y participen en las distintas expresiones culturales. }\end{array}$ & $15.15 \%$ & $(5 / 33)$ \\
\hline $\begin{array}{l}\text { Sean capaces de leer, comprender, emplear, reflexionar e interesarse } \\
\text { en diversos tipos de textos, con el fin de ampliar sus conocimientos } \\
\text { y lograr sus objetivos personales. }\end{array}$ & $12.12 \%$ & $(4 / 33)$ \\
\hline
\end{tabular}

Tabla 8. Análisis de datos clasificados como otros

\begin{tabular}{|l|l|}
\hline PPGUAY02 & Promover la lectura y la escritura de textos. \\
\hline PPCAB05 & Que utilice la lengua materna. \\
\hline PPCAB04 & Que el alumno adquiera el hábito de la lectura. \\
\hline PPGUAY06 & Participe en acuerdos de su nivel y se sienta parte de una sociedad. \\
\hline PPGUAY03 & Que el alumno sea autónomo. \\
\hline PPNOG01 & Que el alumno haga uso de las fuentes de información (TICs). \\
\hline PPHMO06 & Conocimientos técnicos. \\
\hline PPNOG08 & Razonamiento lógico-matemático. \\
\hline PPNOG08 & Diseño y aplicación de estrategias para articular lo emocional y lo cognitivo. \\
\hline
\end{tabular}

Tabla 9. Ejemplos de enunciaciones ajenas a propósitos de la enseñanza del español en educación primaria y educación básica

\section{DISCUSIÓN}

Los procesos de enseñanza y de aprendizaje son complejos puesto que intervienen múltiples factores (como cognitivos, emocionales, sociales). Sin embargo, la claridad en la declaración y en el conocimiento de los propósitos de la enseñanza del español en educación primaria por el docente favorece el diseño y la implementación de estrategias didácticas con las que se promueven aprendizajes significativos del contenido disciplinar, con ello se crean

Noriega Jacob doi: https://doi.org/10.36799/el.v2i1.52 Volumen 2, número 1, Año 2017, ISSN: $2448-5942$ 
ambientes de enseñanza y de aprendizaje que permitan, en la medida de lo posible, atender los posibles factores que se involucran en estos procesos.

La enseñanza de la lengua implica apoyar a los niños para que conozcan los diferentes géneros de texto y que tengan conciencia de las diferencias pragmáticas, discursivas y gramaticales que cada texto requiere. El proceso de enseñanza de la lengua es un espacio de reflexión metalingüística con la que se desarrolla la capacidad de análisis, interpretación y razonamiento a través de la lengua. Esto desde las enunciaciones del grupo de los profesores de primaria respecto a los propósitos de la enseñanza del español queda poco considerado, puesto que gran parte de las enunciaciones refieren a propósitos de la educación básica (véase tabla 7 y tabla 8), así como, a aspectos genéricos del proceso de enseñanza y de aprendizaje (véase tabla 8 y tabla 9). Además, los docentes mencionan la enseñanza y el aprendizaje de la escritura y la lectura sin declarar el conocimiento de los géneros textuales (véase tabla 1 y tabla 2), así como la conciencia y diferencia de la pragmática, discursiva y gramatical que cada texto necesita para ser producido o comprendido. Precisamente a través de este conocimiento y conciencia lingüística el niño inicia el proceso de aprendizaje de la lengua escrita con el que interactúa y/o interactuará dentro de la comunidad de la cultura escrita.

El método utilizado para la recolección de datos, redacción libre de propósitos para la enseñanza del español en educación primaria, buscaba tener una aproximación de los conocimientos que los docentes tienen sobre el tema. Con ello se pretendía observar si el participante del estudio señalaba cuestiones sobre la intencionalidad y motivaciones para la enseñanza, aprendizaje y práctica de la lengua escrita. Si bien es cierto, los datos muestran un panorama sobre los conocimientos de los profesores participantes en relación a los propósitos de la enseñanza del español en educación primaria, a partir del análisis de datos se advierte la importancia de profundizar con otros métodos sobre la inclusión de la intencionalidad de la enseñanza de la lengua; puesto que en este panorama se percata que no se muestra de manera clara y precisa. La declaración de propósitos sin intencionalidad tiene consecuencias en el diseño e implementación de estrategias didácticas, con las que el niño podría tener poca claridad sobre el aprendizaje de la lengua. Incluso, sin la intencionalidad de la enseñanza de la lengua se promueve momentos de simulación de aprendizaje con los que el niño difícilmente encuentra sentido de la utilidad de la lengua en la sociedad de una manera concreta pero principalmente 
real. Las prácticas docentes deben ser centradas en situaciones cercanas a la realidad, donde el niño interactúe y utilice la lengua con fines sociocomunicativos.

En educación primaria se espera que el docente por medio de los propósitos diseñe ambientes de aprendizaje de la lengua y de prácticas sociocomunicativas, con los que el niño logre niveles de dominio de la lengua (ejecutivo, funcional, instrumental y epistemológico). Sin embargo, a partir de los datos se observa que muy probable estos ambientes promueven principalmente el dominio de la lengua en niveles ejecutivos y funcionales. En este sentido, se vuelve un reto para las prácticas docentes al diseñar situaciones y estrategias didácticas con las que el niño desde los primeros años de escolarización domine los demás niveles de la lengua a partir de los propósitos de la enseñanza del español. Enseñar español en educación primaria es más que enseñar a leer y a escribir; en realidad es un espacio donde el niño, además de desarrollar sus competencias lingüísticas, se aproxima al uso de los textos para diferentes fines, conoce su lengua e interactúa con el lenguaje.

En el programa de español de primaria (SEP, 2011) se plantean acciones generales sobre el papel del docente y el trabajo en el aula, que deben: generar espacios de análisis y reflexión, modelar diversas estrategias de lectura que apoyen a la comprensión de textos de los niños, apoyar a los estudiantes en sus procesos de escritura y de lectura, así como, propiciar espacios de discusión entre los alumnos. Sin embargo, sin la precisión de los propósitos de la enseñanza del español en educación primaria estas acciones que el docente debe realizar difícilmente se logran de manera óptima.

Los datos muestran una necesidad urgente de acciones con las que el profesor tenga mayor precisión sobre los propósitos de la enseñanza de la lengua en educación primaria. Las evaluaciones en las que el profesor mexicano participa deja de lado el conocimiento disciplinar de la lengua y curricular con el que día a día trabaja con los niños, la ausencia de esta información muestra un panorama poco claro para la creación de estrategias que tengan por objetivo principal la mejora educativa del país. No obstante, la exploración que se presenta en este artículo evidencia que se requiere abrir espacios donde el profesor reflexione sobre aspectos disciplinares que intervienen en el proceso de aprendizaje de la lengua y sobre cuestiones curriculares. 


\section{BIBLIOGRAFÍA}

Arriaga, Marx. ¿Cómo se comenta la literatura en primaria? Análisis comparativo entre las expectativas planteadas en el Plan de Estudio 2011 y las actividades desarrolladas en los libros de textos oficiales. Reflexiones a partir de la experiencia. Literatura y pedagogía. México: Ediciones Eón, 2014.

Birello, Marilisa y María Rosa Gil. "Creencias sobre la enseñanza de la composición escrita de la gramática de estudiantes de grado de educación primaria y de educación infantil". Tejuelo, Monográfico (2014): 11-26. 5 de enero de 2017. doi:

https://dialnet.unirioja.es/descarga/articulo/5385983.pdf.

Caldera, Reina y Alexis Bermúdez. "Alfabetización académica: comprensión y producción de textos". Educare. 11.37 (2007): 247-256. 5 de enero de 2017. doi:

http:/ / www.redalyc.org/articulo.oa?id=35603710

Carrasco, Alma. "La escuela puede enseñar estrategias de lectura y promover su regular empleo". Revista Mexicana de Investigación Educativa. 8.17 (2003):129-142. 5 de enero de 2017. doi: http://www.redalyc.org/pdf/140/14001708.pdf

Cassany, Daniel. Cocina de la escritura. Barcelona: Anagrama, 1996.

Cassany, Daniel. Construir la escritura. España: Paidós, 1999.

Cassany, Daniel; Marta Luna y Glòria Sanz. Enseñar lengua. México: Paidós, 2007.

Cassany, Daniel. Para ser letrados. Voces y miradas sobre la lectura. España: Paidós, 2009.

Cassidy, Smith y James Bauman. "How to incorporate comprehension monitoring strategies into basal reader instruction". The reading teacher. 40.1 (1986): 28-31. 3 de enero del 2017. doi: https://dialnet.unirioja.es/descarga/articulo/126155.pdf

Cerrillo, Pedro. Sobre lectura, literatura y educación. México: Porrúa, 2010.

Consejo Nacional para la Cultura y las Artes. Encuesta nacional de lectura. México: CONCACULTA, 2006.

Córdova, Doris; Karla Ochoa y Mirna Rizk. "Concepciones sobre la enseñanza de la lectura en un grupo de docentes". Investigación y Postgrado. 1(2009): 159-187.

Noriega Jacob doi: https://doi.org/10.36799/el.v2i1.52 Volumen 2, número 1, Año 2017, ISSN: 2448-5942 
Dubois, María. Textos en contexto 7. Sobre lectura, escritura... y algo más. Argentina: Lectura y Vida, 2006.

Ferreiro, Emilia. Alfabetización. Teoría y práctica. México: Siglo XXI, 2008. Ferreiro, Emilia. Pasado y presente de los verbos leer y escribir. Argentina: Fondo de Cultura Económica, 2012.

Fons, Monserrat. Leery escribirpara vivir. Alfabetización inicialy uso real de la lengua escrita en la escuela. España: Graó, 2007.

Golder, Caroline y Daniel Goanac'h. Leer y comprender. Psicología de la lectura. México: Siglo XXI, 2003.

González, Antonio. Estrategias de comprensión lectora. Aplicación en el aula. España: Editorial Síntesis, 2004.

Gónzalez, Soledad; Paula Solano; Julio Gónzalez-Pineda y José Núñez. “Autorregulación del aprendizaje a partir de textos”. Revista Galego-Portycesa de Psicoloxia e Educación, 11 (2004): 111-127. 16 de octubre del 2011. doi:

https:/ /dialnet.unirioja.es/servlet/articulo?codigo $=1038594$

Herrera, Myriam; Eleonora Varela; Isabel Rueda; Sonia Pelayes; María Reinoso, y Valeria Quiroga. "Evaluación de la educación. Factores que inciden en la comprensión lectora". Congreso Iberoamericano de Educación (2010). 28 de diciembre del 2016. doi:

http://www.chubut.edu.ar/descargas/secundaria/congreso/EVALUACION/R1677_Herrera.pdf

Lerner, Delia. "Lectura y escritura. Apuntes desde la perspectiva curricular." Textos en contextos 4. La escuela y la formación de lectores y escritores. Argentina: Asociación Internacional de Lectura. Lectura y Vida, 2004.

Mayorga, Liliana. "La lectura como estrategia de trabajo en el aula". Revista de docencia universitaria, 6.1 (2005): 232-247. 7 de septiembre de 2011. doi: http://revistas.uis.edu.co/index.php/revistadocencia/article/view/2144

Olson, David y Nancy Torrance. (comps.). Cultura escrita y oralidad. España: Gedisa, 1998.

Peña, Josefina. Las estrategias de lectura: su utilización en el aula. Educare, 4.11 (2000): 159-163. 27 de septiembre de 2011. doi: http://www.redalyc.org/pdf/356/35601104.pdf 
Peredo, María. Lectura y vida cotidiana. Por qué y para qué leen los adultos. México: Paidós, 2005.

Peredo, María. "Las estrategias de localización de información en textos argumentativos que utilizan los estudiantes de bachillerato". Revista Iberoaméricana de Educación, 45.5 (2008). 30 de septiembre de 2011. doi: http://rieoei.org/2300.htm

Secretaría de Educación Pública. Programa de estudios 2011. Guía para el maestro. Educación Básica. Primaria. Tercer grado. México: SEP, 2011.

Serafini, María. Cómo se estudia. La organización del trabajo intelectual. México: Paidós, 2004.

Silvestri, Adriana. "La formulación de preguntas para la comprensión de textos: Estudio experimental”. Revista Signos, 36.62 (2006): 493-510, 21 de septiembre del 2011. doi: http://www.scielo.cl/scielo.php?script=sci_arttext\&pid=S0718-09342006000300008

Tierney, Robert y Jonh Readence. Reading strategies and practices: a compendium. Estados Unidos de América: Allyn \& Bacon Ed., 2000.

Wegnann, Brenda y Miki Knezevie. Mosaic 1 Reading. Estados Unidos de América: McGrawwHill, 2007.

"Estadísticas 2015”. Gob.mx. Secretaría de Educación Pública, 2017. 6 de enero del 2017. http://planea.sep.gob.mx/ba/estadisticas_2015/

\section{AGRADECIMIENTOS}

El Centro Regional de Formación Docente e Investigación Educativa del Estado de Sonora por el financiamiento para la realización del proyecto de investigación "Análisis de las prácticas docentes para la enseñanza de la escritura y la comprensión de textos”, así como a su personal que gestionó y apoyó durante la fase operativa.

La Dirección General de Educación Primaria del Estado de Sonora y la Dirección General de Educación Secundaria del Estado de Sonora, así como a la estructura de coordinaciones de primaria y secundaria que gestionaron la fase operativa del proyecto de investigación "Análisis de las prácticas docentes para la enseñanza de la escritura y la comprensión de textos”.

Noriega Jacob doi: https://doi.org/10.36799/el.v2i1.52 Volumen 2, número 1, Año 2017, ISSN: 2448-5942 
Los profesores de primaria y secundaria que participaron en el proyecto de investigación "Análisis de las prácticas docentes para la enseñanza de la escritura y la comprensión de textos". 